\section{The TCT motif, a key component of an RNA polymerase II transcription system for the translational machinery}

\author{
Trevor J. Parry, ${ }^{1}$ Joshua W.M. Theisen, ${ }^{1}$ \\ Jer-Yuan Hsu, ${ }^{1}$ Yuan-Liang Wang, ${ }^{1}$ \\ David L. Corcoran, ${ }^{2}$ Moriah Eustice, ${ }^{1}$ \\ Uwe Ohler, ${ }^{2,3,4}$ and James T. Kadonaga ${ }^{1,5}$
}

${ }^{1}$ Section of Molecular Biology, University of California at San Diego, La Jolla, California 92093, USA ${ }^{2}$ Institute for Genome Sciences and Policy, Duke University Medical Center, Durham, North Carolina 27708, USA; ${ }^{3}$ Department of Biostatistics and Bioinformatics, Duke University Medical Center, Durham, North Carolina 27708, USA; ${ }^{4}$ Department of Computer Science, Duke University Medical Center, Durham, North Carolina 27708, USA

The TCT motif (polypyrimidine initiator) encompasses the transcription start site of nearly all ribosomal protein genes in Drosophila and mammals. The TCT motif is required for transcription of ribosomal protein gene promoters. The TCT element resembles the Inr (initiator), but is not recognized by TFIID and cannot function in lieu of an Inr. However, a single T-to-A substitution converts the TCT element into a functionally active Inr. Thus, the TCT motif is a novel transcriptional element that is distinct from the Inr. These findings reveal a specialized TCT-based transcription system that is directed toward the synthesis of ribosomal proteins.

Supplemental material is available at http://www.genesdev.org.

Received May 21, 2010; revised version accepted July 30, 2010.

The RNA polymerase II core promoter is a structurally and functionally diverse element that is an important component of the regulation of gene expression (for example, see Smale and Kadonaga 2003; D'Alessio et al. 2009; Juven-Gershon and Kadonaga 2010; Ohler and Wasserman 2010). The core promoter is generally defined as the stretch of DNA that directs the initiation of transcription. With focused (also known as "narrow peak") promoters in which transcription initiates at a single site or in a narrow cluster of sites, the core promoter typically spans from about -40 to +40 relative to the +1 start site. These core promoters are modular in structure, and might contain specific sequence elements such as the TATA box, BRE, Inr (initiator), MTE, and DPE

[Keywords: RNA polymerase II; core promoter; TFIID; ribosomal protein genes]

${ }^{5}$ Corresponding author.

E-MAIL jkadonaga@ucsd.edu; FAX (858) 534-0555.

Article published online ahead of print. Article and publication date are online at http://www.genesdev.org/cgi/doi/10.1101/gad.1951110. motifs. There are, however, no universal core promoter elements.

Although core promoters share the common function of directing the initiation of transcription, they can exhibit different transcriptional properties as a consequence of the presence or absence of particular core promoter elements. For instance, Caudal, a sequence-specific enhancer-binding protein, activates transcription preferentially from DPEcontaining promoters relative to TATA-containing promoters (Juven-Gershon et al. 2008). In addition, the presence of the BREu motif in conjunction with the TATA box further suppresses the ability of Caudal to activate transcription. Consistent with differences in transcriptional mechanisms at different core promoters, the basal transcription factors that transcribe TATA-dependent core promoters are not able to mediate the transcription of DPE-dependent core promoters (Lewis et al. 2005). Moreover, factors such as TBP, NC2, and Mot1 differentially influence transcription from TATA-dependent versus DPEdependent core promoters (Willy et al. 2000; Hsu et al. 2008). In a related phenomenon, it has also been found that the factors that mediate basal transcription through a single element, the TATA box, can change upon differentiation of cells (Deato and Tjian 2007; Deato et al. 2008).

Many core promoters appear to lack the more extensively characterized core promoter motifs. We therefore sought to expand our understanding of core promoter elements. These studies led to the analysis of a core promoter motif at the transcription start sites of the Drosophila ribosomal protein (RP) gene family. This conserved sequence was originally found in mammalian RP gene promoters, and has been termed the polypyrimidine initiator (for example, see Perry 2005; Roepcke et al. 2006). Here we investigate the properties of this sequence, which we abbreviated as the TCT motif, based on the sequence of the pyrimidine nucleotides that encompass the $\mathrm{C}+1$ start site. The TCT motif, which spans from -2 to +6 relative to the +1 transcription start site, overlaps with but is functionally distinct from the DNA encoding the $5^{\prime}$-terminal oligopyrimidine tract $\left(5^{\prime}-\mathrm{TOP}\right)$, which is a polypyrimidine stretch in the $5^{\prime}$ end of RP mRNAs that is involved in the regulation of translation (for reviews, see Meyuhas 2000; Hamilton et al. 2006). Specifically, the TCT motif functions in a manner that is parallel to and distinct from the Inr element, and serves as a key component of an RNA polymerase II system that is directed toward the expression of RP genes as well as other genes encoding factors involved in protein synthesis.

\section{Results and Discussion}

\section{$R P$ gene core promoters and the TCT motif}

To identify new sequences that contribute to core promoter activity, we analyzed promoters that appear to lack the BRE, TATA, Inr, MTE, and DPE motifs. With this approach, we sought to find focused promoters that are driven by novel core promoter elements. To this end, we screened core promoter sequences (based on transcription start sites in Drosophila melanogaster) (Ohler et al. 2002; Ahsan et al. 2009) and identified a variety of potential new motif-containing core promoters. We then examined the promoter activity of 50 of these core promoters by in vitro transcription analysis of constructs containing sequences 
from -50 to +50 relative to the transcription start site. The transcriptionally active core promoters included two RP gene promoters: $R p L P 1$ and $R p S 15$ (Fig. 1A). Transcription from these promoters is carried out by RNA polymerase II, as assessed by sensitivity to $4 \mu \mathrm{g} / \mathrm{mL} \alpha$-amanitin.

With regard to the core promoter region, the RP genes are notable because mammalian RP gene promoters have been found to contain conserved sequences that encompass the transcription start site (for example, see Perry 2005; Roepcke et al. 2006). This conserved sequence, YYC+1TYTTYYY (Perry 2005), is known as the polypyrimidine initiator and overlaps with the DNA encoding the $5^{\prime}$-TOP, which is an extended ( 5-15 nucleotides [nt]) oligopyrimidine sequence at the $5^{\prime}$ end of RP mRNAs that is important for translational regulation (for reviews, see Meyuhas 2000; Hamilton et al. 2006). Mutations in the polypyrimidine initiator in the mouse $r p S 16$ promoter were found to result in a change in the site of transcription initiation as well as an increase or decrease in the efficiency of transcription (Hariharan and Perry 1990; Chung and Perry 1991). These studies did not reveal whether the polypyrimidine initiator is a subclass of the canonical Inr or a new element that is functionally distinct from the Inr.

Because of the conservation of the polypyrimidine initiator, as well as the biological importance of ribosome biogenesis, we further investigated the transcriptional properties of Drosophila RP gene core promoters. First, we identified 52 Drosophila RP genes (Nakao et al. 2004) with high-confidence transcription start sites that are identical in two different data sets (Ahsan et al. 2009; Ni et al. 2010). We then aligned the core promoter sequences of these $52 \mathrm{RP}$ genes relative to the predicted $\mathrm{C}+1$ start site, and found a shared sequence (YYC+1TTTYY) (Fig. 1B; Supplemental Table 1) that appears to be a more strictly conserved subset of the mammalian polypyrimidine initiator. Strikingly,

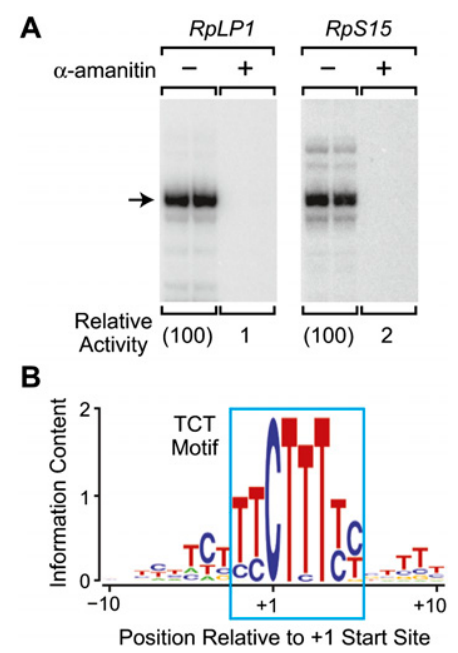

Figure 1. Analysis of core promoters of Drosophila RP genes. $(A)$ Transcription of RP genes by RNA polymerase II. Core promoter constructs containing sequences from -50 to +50 (relative to the $\mathrm{C}+1$ transcription start site) of the RpLP1 and $R p S 15$ genes were subjected to in vitro transcription analysis in the absence or presence of $4 \mu \mathrm{g} / \mathrm{mL} \alpha$-amanitin. The resulting transcripts were detected by primer extension analysis. (B) DNA sequence logo of the TCT motif, which encompasses the transcription start site region of RP genes. The sequence logo was generated as described elsewhere (Schneider and Stephens 1990; Crooks et al. 2004) with the core promoter sequence data in Supplemental Table 1. the $\mathrm{C}+1, \mathrm{~T}+2$, and $\mathrm{T}+4$ are invariant in the 52 Drosophila $\mathrm{RP}$ gene promoters (Supplemental Table 1). Based on the pyrimidine nucleotides that encompass the $\mathrm{C}+1$ start site, we adopted the shorthand notation TCT to denote the polypyrimidine initiator.

In addition to the core promoters with high-confidence start sites (Supplemental Table 1), the TCT motif appears to be present at the predicted transcription start sites of most of the other RP genes (Supplemental Table 2). It is notable, however, that the CCATTT sequences that encompass the predicted transcription start sites in the sta and sop RP genes resemble the Inr consensus of TCA $+1 \mathrm{KTY}$, particularly at the critical CA nucleotides. Thus, the sta and sop core promoters may possess Inr rather than TCT elements.

With human transcription start site data, we analyzed the occurrence of the TCT motif in human RP gene promoters and found a consensus of YC+1TYTYY (Supplemental Table 3), which closely resembles the Drosophila TCT consensus (YYC+1TTTYY). Notably, 48 out of the 50 human RP genes examined contain a six out of seven or seven out of seven match to the human TCT consensus sequence, either in precise alignment with the predicted +1 transcription start site or upon 1- or 2-nt realignment of the start site (Supplemental Table 3B). The similarity of the human and Drosophila consensus sequences suggests that the function of the TCT motif is conserved from Drosophila to humans.

We also investigated whether the TCT motif is present in the core promoter regions of non-RP-encoding genes. To this end, we screened the Drosophila Machibase data set (Ahsan et al. 2009) for well-represented (>400 tags) transcription start sites that possess a seven out of eight or eight out of eight match with the TCT consensus as well as a $\mathrm{C}+1$ nucleotide at the predicted start site (Supplemental Table 4). This analysis revealed that the TCT sequence is present at the core promoters of genes encoding translation initiation and elongation factors (Ef2b, eIF-5A, Int6) eIF3-S6, and eIF3-S8), a tRNA synthetase (Aats-tyr), and a polyA-binding protein $(p A b p)$. These findings suggest that the relation between the TCT motif and translation extends beyond the RPs.

\section{Sequences within the TCT motif are required for transcription of $R P$ gene promoters}

We next sought to determine whether the TCT sequences are important for transcriptional activity. To this end, we carried out systematic mutational analyses of the promoters of three different RP genes: $R p L P 1, R p S 15$, and $R p S 12$. For each promoter, we constructed and analyzed a series of triple-nucleotide substitution mutations from -4 to +11 relative to the $\mathrm{C}+1$ start site (Fig. 2A). Because of the pyrimidine-rich nature of the TCT motif, we mutated the natural sequences to GGG trinucleotides. We avoided the use of adenines to prevent the inadvertent formation of an Inr motif, which has a key $A+1$ nucleotide. In addition, with the $R p L P 1$ and $R p S 15$ promoters, the natural sequences from +6 to +8 are CGG and CGT, respectively; we therefore constructed both GGG and TTT $+6+7+8$ mutant versions of those promoters to ensure that the mutations would alter any potentially important sequences in the +6 to +8 region.

These experiments revealed that mutation of sequences from -1 to +2 as well as from +3 to +5 results in a substantial reduction in transcription of the $R p L P 1$, 
A

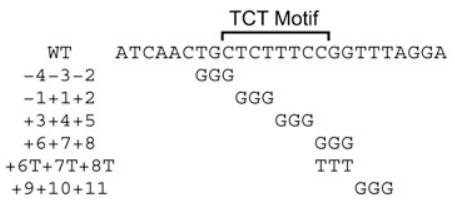

B

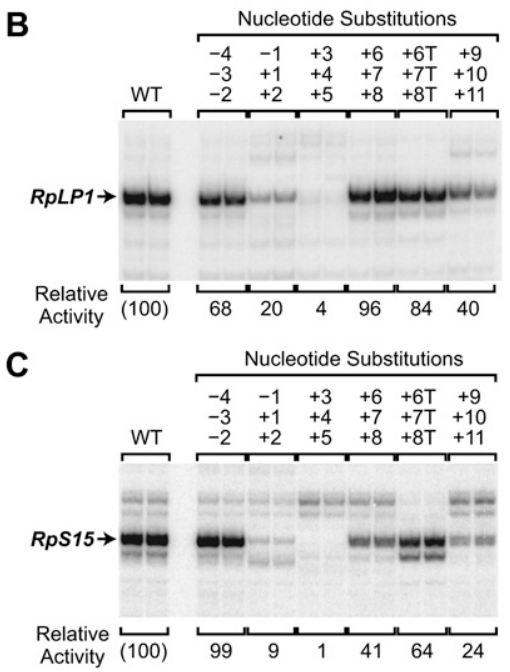

Figure 2. Sequences in the TCT motif are critical for transcription from RP gene core promoters. (A) Diagram of scanning triplenucleotide substitution mutations. The trinucleotides at the specified positions (relative to the $\mathrm{C}+1$ ) were converted to GGG, except as indicated for the $+6 \mathrm{~T}+7 \mathrm{~T}+8 \mathrm{~T}$ mutant promoter. The sequence of the Drosophila RpLP1 core promoter is shown. An analogous series of mutations was constructed with the RpS15 core promoter. $(B, C)$ In vitro transcription analyses of the wild-type and mutant $R p L P 1$ and $R p S 15$ promoters. The transcriptional activity of each mutant promoter is reported relative to that of its cognate wild-type promoter.

RpS15, and RpS12 core promoters (Fig. 2; Supplemental Fig. S1). There is a particularly strong loss of transcriptional activity upon mutation of TCT sequences from positions +3 to +5 . On the other hand, mutation of the highly conserved (Fig. 1B) -1 to +2 sequences resulted in a threefold to 11-fold decrease in transcription, which might be less than expected, perhaps due to contributions from the remaining flanking nucleotides. We also observed a moderate decrease in transcription with the +9 to +11 mutation in the RpLP1 and RpS15 promoters, but not in the RpS12 promoter (Supplemental Fig. S1). Hence, mutation of the +9 to +11 region does not consistently result in a reduction of transcriptional activity. Thus, these findings indicate that the central $6 \mathrm{nt}$ of the TCT motif are most important for transcription of the RP gene core promoters.

\section{Purified TFIID has little or no affinity for the} TCT-dependent RpLP1 core promoter

The core of the TCT motif, YCTTTY, closely resembles the Inr consensus, which is TCAKTY in Drosophila and YYANWYY in humans (for instance, see Smale and Kadonaga 2003; Juven-Gershon et al. 2010). (Although transcription generally initiates from the $\mathrm{A}+1$ in the $\mathrm{Inr}$ [TCA+1KTY] and the C+1 in the TCT motif [YYC+1TTTYY], transcription initiation from the $\mathrm{C}+1$ position of the Inr [i.e., TC+1AKTY] has also been observed with Inrdependent core promoters [for instance, see Lim et al. 2004].) We therefore considered the possibility that the TCT element is related to the Inr.
To test whether the TCT motif could be a subset of the Inr, we carried out DNase I footprinting analyses of wildtype and mutant $(+3+4+5$ GGG) versions of the $R p L P 1$ core promoter with purified TFIID (Supplemental Fig. S2). TFIID is a key factor that binds to the Inr motif (for example, see Kaufmann and Smale 1994; Martinez et al. 1994; Purnell et al. 1994; Verrijzer et al. 1995; Burke and Kadonaga 1996; Emami et al. 1997; Chalkley and Verrijzer 1999; Smale and Kadonaga 2003). As positive controls for the binding of TFIID, we performed DNase I footprinting reactions in parallel with the TATA-dependent $h b \mathrm{P} 2$ core promoter as well as the DPE-dependent $E 74 B$ core promoter. The $R p L P 1, h b P 2$, and $E 74 B$ promoters exhibit similar levels of transcriptional activity in vitro (Supplemental Fig. S2A). As shown in Supplemental Figure S2B, there is little or no interaction of purified TFIID with the wild-type $R p L P 1$ core promoter region under conditions in which distinct TFIID-induced DNase I protection and hypersensitivity is seen with the $h b \mathrm{P} 2$ and $E 74 B$ core promoters. There is a faint hypersensitive site at about +3 in the wild-type but not mutant $R p L P 1$ promoter; however, this slight hypersensitive band is much less distinct than the regions of DNase I protection and hypersensitivity that are seen in the $h b \mathrm{P} 2$ and $E 74 B$ promoters. Thus, TFIID exhibits little or no binding to the TCT-dependent $R p L P 1$ core promoter. These findings suggest that the TCT motif is not a functionally related variant of the Inr.

\section{A single nucleotide can determine whether a sequence functions as a TCT element or an Inr}

We further analyzed the relation between the TCT motif and the Inr by testing whether the TCT element could substitute for the Inr. In these experiments, we used the TATA-dependent adenovirus major late (AdML) core promoter as well as the DPE-dependent Drosophila E74B core promoter. With the AdML promoter, we replaced the Inr (TCACTC) and its flanking two nucleotides (CTCACTCT) with the TCT motif (CTCTTTCC) from the RpLP1 core promoter (Supplemental Table 1). As depicted in Figure 3A, the replacement of the AdML Inr with the TCT sequence results in an $\sim 10$-fold decrease in transcriptional activity to a level that is comparable with that seen upon mutation of the AdML Inr from TCACTC to GTGACA ("mInr" in Fig. 3A). Thus, the TCT element cannot substitute for the Inr in the TATA-dependent AdML core promoter. This finding, which is consistent with the inability of TFIID to bind to the TCT-dependent RpLP1 core promoter (Supplemental Fig. S2), was nevertheless intriguing because of the close sequence similarity between the Inr and TCT motifs. A key difference, however, between the TCT and Inr sequences is the presence of the A+1 nucleotide in the Inr. We therefore generated a single T-to-A substitution in the TCT sequence to give CTCATTCC ("TCA" in Fig. 3A), in which the central $6 \mathrm{nt}$ (TCATTC) correspond to a consensus Inr. This T-to-A substitution in the TCT motif resulted in the restoration of Inr activity. Thus, the TCT motif is distinct from the Inr, and a single nucleotide can determine whether the sequence functions as a TCT motif or an Inr.

To complement the studies with the TATA-dependent AdML core promoter, we analyzed the ability of the TCT motif to substitute for the Inr in the DPE-dependent $E 74 B$ and Antennapedia P2 (Antp P2) core promoters. With the $E 74 B$ promoter (Fig. 3B), replacement of the Inr with the TCT motif results in an $\sim 25$-fold decrease in transcriptional activity to a level that is comparable with that seen with the 


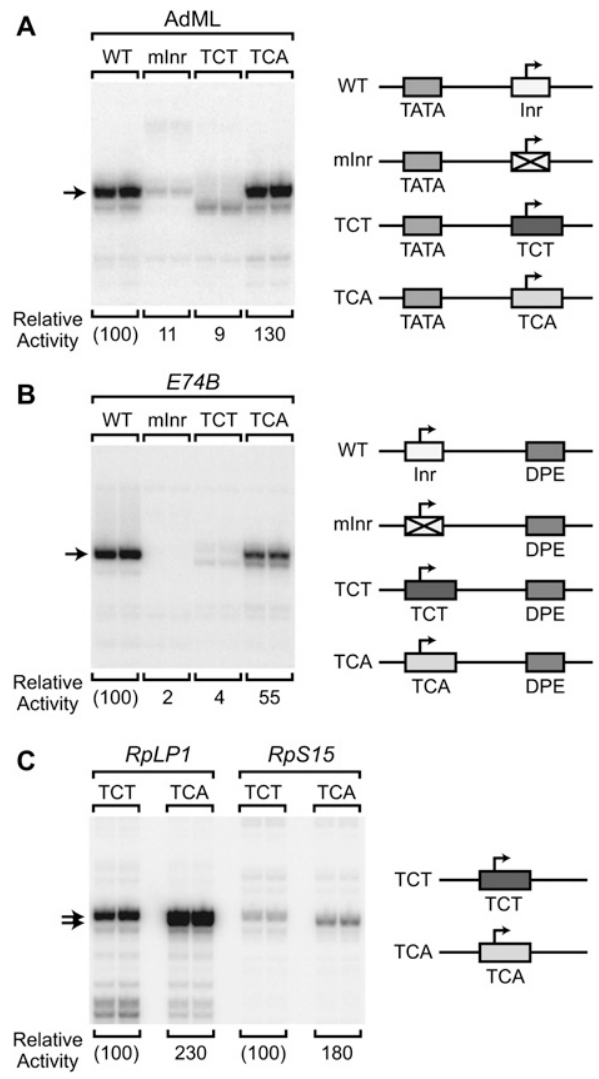

Figure 3. A single T-to-A substitution can convert a TCT element, which does not function with TATA or DPE motifs, into an Inr. $(A)$ The TCT motif does not function with a TATA box in lieu of an Inr. Wild-type and mutant versions of the AdML core promoter were subjected to in vitro transcription analysis. In the mInr promoter the Inr (TCACTC) was mutated to GTGACA. In the TCT promoter, the AdML Inr and flanking nucleotides (CTCACTCT) were replaced with the corresponding TCT sequence (CTCTTTCC) from the RpLP1 core promoter. The TCA promoter contains a T-to-A substitution in the TCT motif (CTCATTCC). The transcriptional activity of each mutant promoter is reported relative to that of the wild-type AdML promoter. $(B)$ The TCT motif does not function with a DPE in lieu of an Inr. Wild-type and mutant versions of the DPE-dependent $E 74 B$ core promoter were generated and analyzed as described in $A$ with the AdML promoter. $(C)$ The TCT-to-TCA mutation in RP gene promoters results in a downstream shift of the transcription start site. The TCT sequence in the TCT motifs of the $R p L P 1$ and $R p S 15$ core promoters were mutated to TCA, and the resulting wild-type and mutant constructs were subjected to in vitro transcription analysis.

mInr mutation. Hence, the TCT motif cannot substitute for the Inr element in the DPE-dependent $E 74 B$ core promoter. Moreover, as seen with the TATA-dependent AdML promoter, we found that a T-to-A substitution in the TCT motif ("TCA"; CTCATTCC) restores (to $55 \%$ of wild-type activity) the transcriptional activity of the DPE-dependent $E 74 B$ promoter. With the Antp P2 promoter (Supplemental Fig. S3), we obtained essentially the same results as with the E74B promoter. Specifically, the TCT motif cannot substitute for the Inr element, and mutation of the TCT sequence to TCA results in a restoration of transcriptional activity.

We additionally tested the effect of the T-to-A substitution in the TCT motif in the context of RP gene promoters. We generated and analyzed "TCA" versions of the Drosophila RpLP1 and RpS15 core promoters. As shown in Figure 3C, the T-to-A substitution results in a shift in the site of transcription initiation to a downstream start site, as well as a modest (about twofold) increase in transcriptional activity. A similar downstream shift in the transcription start site was observed with a C-to-A substitution (which converted a TCC sequence to TCA) in the mouse rpS16 promoter (Hariharan and Perry 1990; Chung and Perry 1991). These results, in combination with the findings described above, suggest that the T-to-A substitution in the RP gene promoters results in the conversion of the promoter from a TCT-based transcription system to an Inr-based system.

To investigate the basis for the transcriptional effects, we performed DNase I footprinting analyses of these constructs with purified TFIID (Fig. 4). Consistent with the results shown in Supplemental Figure S2, we found that TFIID binds to the Inr-containing AdML and $E 74 B$ core promoters but not to the TCT-containing RpLP1 core promoter. With the AdML and $E 74 B$ promoters, substitution of the Inr with a TCT motif results in a nearly complete loss of TFIID binding, even in the presence of a TATA box (AdML) or DPE (E74B). However, the binding of TFIID to these promoters is restored upon mutation of the TCT sequence to TCA (CTCTTTCC to CTCATTCC). In addition, substitution of the TCT motif in the RpLP1 promoter with the TCA sequence leads to an increase in the binding of TFIID. These findings reveal that the TCT-to-TCA singlenucleotide substitution converts the TCT motif, which is not a binding site for TFIID, into an Inr, which is a TFIID recognition sequence.

Thus, the TCT motif is a core promoter element that is functionally distinct from the Inr (Supplemental Fig. S4). Unlike the Inr, the TCT motif is not a recognition site for TFIID and does not promote transcription in conjunction with the TATA or DPE core promoter motifs. However, a single T-to-A substitution of the TCT trinucleotide into TCA can convert a TCT motif into an active Inr element.

\section{A TCT-based RNA polymerase II system for the transcription of $R P$ genes}

The findings of this study suggest the existence of a TCTbased RNA polymerase II system for the transcription of RP genes. The TCT motif, which spans from -2 to +6 relative to the +1 start site, overlaps with and extends upstream of the DNA encoding the $5^{\prime}$-TOP, which regulates the translation of RP mRNAs. It thus appears that there are dual transcriptional and translational functions of the polypyrimidine sequences at the $5^{\prime}$ end of the RP genes.

The TCT motif is present in the same position (relative to the transcription start site) in nearly all Drosophila RP genes (Supplemental Tables 1,2) as well as in at least 48 human RP genes (Supplemental Table 3). In addition, the core promoters of some genes encoding translation initiation and elongation factors contain TCT elements (Supplemental Table 4). Scanning mutational analyses of three different RP gene promoters showed that the highly conserved, central $6 \mathrm{nt}$ of the TCT motif (YCTTTY) are the most important region for transcriptional activity (Fig. 2; Supplemental Fig. S1). Notably, this central core of the TCT element closely resembles the Inr consensus, which is TCAKTY in Drosophila. Yet, even though the sequence of the TCT motif is nearly identical to that of the Inr, the TCT motif is unable to replace the Inr in TATA-dependent or DPE-dependent core promoters (Fig. 3A,B) and is not a recognition site for the binding of purified 


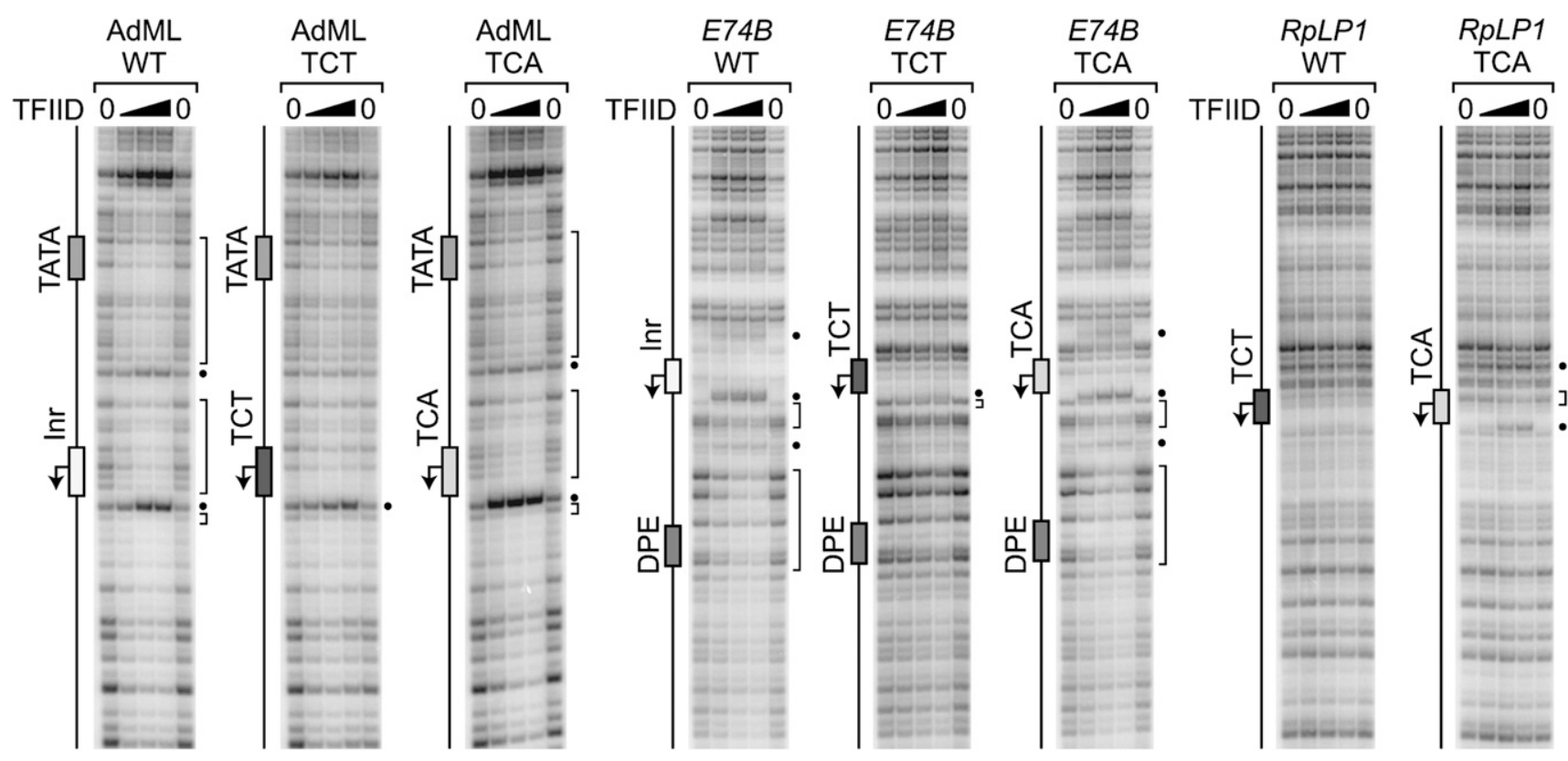

Figure 4. A single T-to-A substitution in the TCT motif substantially increases the affinity of TFIID binding to the core promoter. DNase I footprinting analyses were carried out with purified Drosophila TFIID. With the AdML and E74B promoter constructs, the five footprint reactions contained $0,30,60,90$, and 0 ng of TFIID. With the RpLP1 promoter constructs, the reactions contained 0, 15, 30, 45, and 0 ng of TFIID. Regions of DNase I protection and hypersensitivity are indicated by brackets and solid dots, respectively.

TFIID (Fig. 4; Supplemental Fig. S2). However, a single T-to-A substitution in the TCT element that changes the TCT trinucleotide to TCA converts the TCT element into an Inr (Figs. 3, 4; Supplemental S3). These findings reveal that the TCT-based RNA polymerase II transcription system is distinct from the widely used Inr-based transcription system.

The TCT motif is an example of a core promoter element that is involved in the regulation of a network of genes. It has also been seen, for instance, that the DPE core promoter motif is used widely among the Drosophila homeotic genes, and that the Caudal protein is a DPEspecific transcriptional activator (Juven-Gershon et al. 2008). The TCT motif appears to be present in the core promoters of RP genes from Drosophila to humans, but does not appear to be present in the yeast Saccharomyces cerevisiae. Therefore, the TCT motif may be used predominantly in metazoans. In Drosophila, the TCT element is present in perhaps $\sim 120$ core promoters (including the RP gene promoters); hence, it is a rare sequence motif. It is therefore useful to consider that there may be other rare but important core promoter elements that have not yet been identified.

Protein synthesis is one of the most critical biochemical processes in life. The specialized TCT-based transcription system for the synthesis of RPs is, in many respects, complementary to the specialized RNA polymerase I and RNA polymerase III transcription systems for the production of rRNAs and tRNAs. In the case of the RPs, it is essential for the cell to achieve high levels of expression of the RP genes as well as to coordinate the relative amounts of each of the proteins. The analysis of the TCT-based transcription system should provide new and important insights into the means by which transcription is regulated to achieve optimal translation in metazoans.

\section{Materials and methods}

\section{In vitro transcription analysis}

Double-stranded oligonucleotides comprising sequences from -50 to +50 of the core promoters (relative to $\mathrm{C}+1$ in the TCT motif or to $\mathrm{A}+1$ in the Inr), except for AdML (which contained sequences from -33 to +10 relative to the $\mathrm{A}+1$ in the $\mathrm{Inr}$ ), were inserted into the PstI and $\mathrm{XbaI}$ sites of pUC119. The sequences of mutant versions of these promoters are specified in the figures and figure legends. In vitro transcription reactions were carried out as described previously (Wampler et al. 1990) by using 250 ng of supercoiled DNA templates with Drosophila high-salt nuclear extracts (Soeller et al. 1988). The resulting transcripts were subjected to primer extension analysis with an M13 reverse-sequencing primer (AGCG GATAACAATTTCACACAGGA). Quantitation of reverse transcription products was carried out with a Typhoon PhosphorImager (GE Health Sciences). All experiments were carried out a minimum of two (but usually three or more) independent times to ensure reproducibility of the data.

\section{DNase I footprinting assays}

DNase I footprinting probes were prepared by PCR amplification of each promoter with unlabeled M13 universal primer (located upstream of the transcription start site) and 5' -32P-labeled M13 reverse-sequencing primer (located downstream from the start site). The PCR products were purified by electrophoresis on a nondenaturing $5 \%$ polyacrylamide gel. TFIID was purified to near homogeneity by sequential sequence-specific DNA affinity chromatography and anti-Flag immunoaffinity chromatography of nuclear extracts derived from Drosophila S2 cells containing Flagtagged TBP (Theisen et al. 2010). DNase I footprinting reactions were performed as described previously (Burke and Kadonaga 1996).

\section{Acknowledgments}

Dedicated to Robert Tjian on the occasion of his 60th birthday. We thank Tamar Juven-Gershon, Zhengjian Zhang, Timur Yusufzai, Sharon Torigoe, and James Gucwa for critical reading of the manuscript. We are grateful to Robert P. Perry (Fox Chase Cancer Center, Philadelphia, PA) for stimulating our interest in RP gene transcription. This work was supported by 
grants from the National Institutes of Health to J.T.K. (R01 GM041249) and U.O. (R01 HG004065).

\section{References}

Ahsan B, Saito TL, Hashimoto S, Muramatsu K, Tsuda M, Sasaki A, Matsushima K, Aigaki T, Morishita S. 2009. Machibase: A Drosophila melanogaster 5 '-end mRNA transcription database. Nucleic Acids Res 37: D49-D53. doi: 10.1093/nar/gkn694.

Burke TW, Kadonaga JT. 1996. Drosophila TFIID binds to a conserved downstream basal promoter element that is present in many TATAbox-deficient promoters. Genes Dev 10: 711-724.

Chalkley GE, Verrijzer CP. 1999. DNA binding site selection by RNA polymerase II TAFs: A $\mathrm{TAF}_{\mathrm{II}} 250-\mathrm{TAF}_{\mathrm{II}} 150$ complex recognizes the Initiator. $E M B O J$ 18: 4835-4845.

Chung S, Perry RP. 1991. Cell-free transcription of a mouse ribosomalprotein-encoding gene: The effects of promoter mutations. Gene 100: 173-180.

Crooks GE, Hon G, Chandonia J-M, Brenner SE. 2004. WebLogo: A sequence logo generator. Genome Res 14: 1188-1190.

D'Alessio JA, Wright JK, Tjian R. 2009. Shifting players and paradigms in cell-specific transcription. Mol Cell 36: 924-931.

Deato MDE, Tjian R. 2007. Switching of the core transcription machinery during myogenesis. Genes Dev 21: 2137-2149.

Deato MDE, Marr MT II, Sottero T, Inouye C, Hu P, Tjian R. 2008. MyoD targets TAF3/TRF3 to activate myogenin transcription. Mol Cell 32: 96-105.

Emami KH, Jain A, Smale ST. 1997. Mechanism of synergy between TATA and initiator: Synergistic binding of TFIID following a putative TFIIA-induced isomerization. Genes Dev 11: 3007-3019.

Hamilton TL, Stoneley M, Spriggs KA, Bushell M. 2006. TOPs and their regulation. Biochem Soc Trans 34: 12-16.

Hariharan N, Perry RP. 1990. Functional dissection of a mouse ribosomal protein promoter: Significance of the polypyrimidine initiator and an element in the TATA-box region. Proc Natl Acad Sci 87: $1526-1530$.

Hsu JY, Juven-Gershon T, Marr MT II, Wright KJ, Tjian R, Kadonaga JT. 2008. TBP, Mot1, and NC2 establish a regulatory circuit that controls DPE-dependent versus TATA-dependent transcription. Genes Dev 22: 2353-2358.

Juven-Gershon T, Kadonaga JT. 2010. Regulation of gene expression via the core promoter and the basal transcriptional machinery. Dev Biol 339: 225-229.

Juven-Gershon T, Hsu JY, Kadonaga JT. 2008. Caudal, a key developmental regulator, is a DPE-specific transcription factor. Genes Dev 22. 2823-2830.

Kaufmann J, Smale ST. 1994. Direct recognition of initiator elements by a component of the transcription factor IID complex. Genes Dev 8: 821-829.

Lewis BA, Sims RJ III, Lane WS, Reinberg D. 2005. Functional characterization of core promoter elements: DPE-specific transcription requires the protein kinase $\mathrm{CK} 2$ and the PC4 coactivator. Mol Cell 18: 471-481.

Lim CY, Santoso B, Boulay T, Dong E, Ohler U, Kadonaga JT. 2004. The MTE, a new core promoter element for transcription by RNA polymerase II. Genes Dev 18: 1606-1617.

Martinez E, Chiang CM, Ge H, Roeder RG. 1994. TATA-binding proteinassociated factor(s) in TFIID function through the initiator to direct basal transcription from a TATA-less class II promoter. EMBO $J$ 13: 3115-3126.

Meyuhas O. 2000. Synthesis of the translational apparatus is regulated at the translational level. Eur J Biochem 267: 6321-6330.

Nakao A, Yoshihama M, Kenmochi N. 2004. RPG: The ribosomal protein gene database. Nucleic Acids Res 32: D168-D170. doi: 10.1093/nar/ gkh004.

Ni T, Corcoran DL, Rach EA, Song S, Spana EP, Gao Y, Ohler U, Zhu J. 2010. A paired-end sequencing strategy to map the complex landscape of transcription initiation. Nat Methods 7: 521-527.

Ohler U, Wassarman DA. 2010. Promoting developmental transcription. Development 137: 15-26.

Ohler U, Liao GC, Niemann H, Rubin GM. 2002. Computational analysis of core promoters in the Drosophila genome. Genome Biol 3: research0087. doi: 10.1186/gb-2002-3-12-research0087.
Perry RP. 2005. The architecture of mammalian ribosomal protein promoters. BMC Evol Biol 5: 15. doi: 10.1186/1471-2148-5-15.

Purnell BA, Emanuel PA, Gilmour DS. 1994. TFIID sequence recognition of the initiator and sequences farther downstream in Drosophila class II genes. Genes Dev 8: 830-842.

Roepcke S, Zhi D, Vingron M, Arndt PF. 2006. Identification of highly specific localized sequence motifs in human ribosomal protein gene promoters. Gene 365: 48-56.

Schneider TD, Stephens RM. 1990. Sequence logos: A new way to display consensus sequences. Nucleic Acids Res 18: 6097-6100.

Smale ST, Kadonaga JT. 2003. The RNA polymerase II core promoter. Annu Rev Biochem 72: 449-479.

Soeller WC, Poole SJ, Kornberg T. 1988. In vitro transcription of the Drosophila engrailed gene. Genes Dev 2: 68-81.

Theisen JWM, Lim CY, Kadonaga JT. 2010. Three key subregions contribute to the function of the downstream RNA polymerase II core promoter. Mol Cell Biol 30: 3471-3479.

Verrijzer CP, Chen JL, Yokomori K, Tjian R. 1995. Binding of TAFs to core elements directs promoter selectivity by RNA polymerase II. Cell 81: 1115-1125.

Wampler SL, Tyree CM, Kadonaga JT. 1990. Fractionation of the general RNA polymerase II transcription factors from Drosophila embryos. J Biol Chem 265: 21223-21231.

Willy PJ, Kobayashi R, Kadonaga JT. 2000. A basal transcription factor that activates or represses transcription. Science 290: 982-984. 


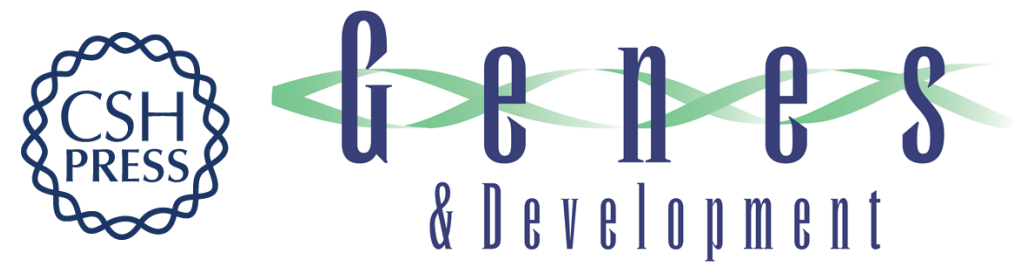

\section{The TCT motif, a key component of an RNA polymerase II transcription system for the translational machinery}

Trevor J. Parry, Joshua W.M. Theisen, Jer-Yuan Hsu, et al.

Genes Dev. 2010, 24: originally published online August 27, 2010

Access the most recent version at doi:10.1101/gad.1951110

\section{Supplemental http://genesdev.cshlp.org/content/suppl/2010/08/19/gad.1951110.DC1 \\ Material}

References This article cites 33 articles, 16 of which can be accessed free at:

http://genesdev.cshlp.org/content/24/18/2013.full.html\#ref-list-1

License

Email Alerting

Receive free email alerts when new articles cite this article - sign up in the box at the top

Service

right corner of the article or click here.

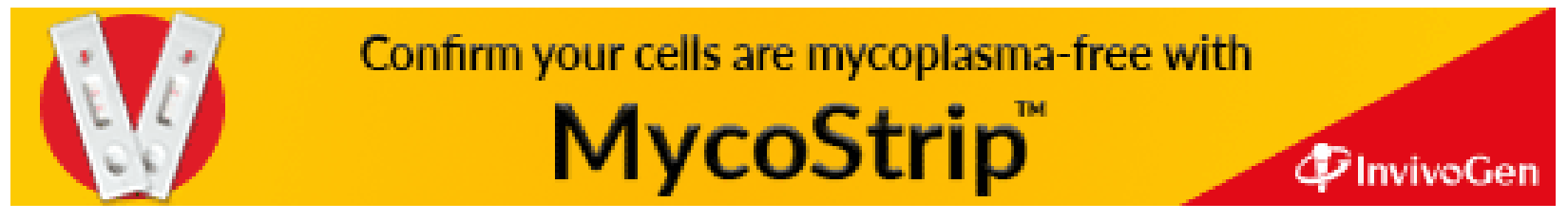

